\title{
InGaAsSb/AIGaAsSb Heterojunction Phototransistors for Infrared Applications
}

\author{
Tamer F. Refaat ${ }^{1}$, M. Nurul Abedin ${ }^{2}$, Oleg V. Sulima ${ }^{3}$, Syed Ismail ${ }^{2}$ and Upendra N. Singh ${ }^{2}$ \\ ${ }^{1}$ Old Dominion University, Norfolk, VA 23529 \\ ${ }^{2}$ NASA Langley Research Center, Hampton, VA 23681 \\ ${ }^{3}$ University of Delaware, Newark, DE 19716
}

\begin{abstract}
High quality infrared (IR) quantum detectors are important for several applications, such as atmospheric remote sensing, chemical detection and absorption spectroscopy. Although several IR detectors are commercially available, with different materials and structures, they provide limited performance regarding the signal-to-noise ratio and the corresponding minimum detectable signal. InGaAsSb/AlGaAsSb heterojunction based phototransistors show strong potential for developing IR sensors with improved performance.

In this paper, the performance of a novel npn InGaAsSb/AlGaAsSb heterojunction phototransistor is presented. This performance study is based on experimental characterization of the device dark current, noise and spectral response. Detectivity of $1.7 \times 10^{9} \mathrm{cmHz}^{1 / 2} / \mathrm{W}$ at $2-\mu \mathrm{m}$ was obtained at $100^{\circ} \mathrm{C}$ temperature and $2 \mathrm{~V}$ bias voltage. This corresponds to a responsivity of $94.7 \mathrm{~A} / \mathrm{W}$ and an internal gain of 156 with about $37.7 \%$ quantum efficiency. Reducing the temperature to $-30^{\circ} \mathrm{C}$ allows to increase the bias to $3 \mathrm{~V}$ and enhance the detectivity to $8.7 \times 10^{10} \mathrm{cmHz}^{1 / 2} / \mathrm{W}$ at the same wavelength, which corresponds to a responsivity of $386.5 \mathrm{~A} / \mathrm{W}$ and an internal gain of 288.2 with about $83.3 \%$ quantum efficiency. The device impulse response and linearity, including the corresponding dynamic range, also are presented. Impulse response analysis indicated a settling time of about $1.1 \mu \mathrm{s}$ at $2 \mathrm{~V}$ and $100^{\circ} \mathrm{C}$, while linearity measurements indicated a constant responsivity in the radiation intensity range of $1.6 \times 10^{-7} \mathrm{~W} / \mathrm{cm}^{2}$ and $31.6 \mathrm{~mW} / \mathrm{cm}^{2}$.
\end{abstract}

Keywords: Phototransistors, IR Detectors, Characterization, InGaAsSb, AlGaAsSb, linearity, impulse response

\section{INTRODUCTION}

III-V compound materials are suitable for fabricating optoelectronic devices in the near and mid-infrared wavelength range. The availability of binary substrates, such as InAs and GaSb, allows growth of multilayer homo and hetero-structures, where lattice matched ternary and quaternary layers could be tailored to detect wavelengths in the range of 0.8 to $4 \mu \mathrm{m}^{1}$. Such detectors are useful for several applications, including atmospheric remote sensing and optical communications. Even binary III-V compound detectors, such as InAs and InSb, indicated reasonable performance in the mid-infrared range. Commercially, these detectors are available either in a photoconductive or photovoltaic configuration. They have sensitivity at wavelengths as far as 3.5 and $6.9 \mu \mathrm{m}$, respectively but with limited performance. The detectivity $\left(\mathrm{D}^{*}\right)$ of these devices can reach $10^{11} \mathrm{cmHz}^{1 / 2} / \mathrm{W}$ by cooling down using liquid nitrogen, which increases the complexity of their applied systems $\mathrm{s}^{2,3}$. With a maximum $\mathrm{D}^{*}$ close to $10^{12} \mathrm{cmHz} / 1 / 2 / \mathrm{W}$ and diameter as large as $3 \mathrm{~mm}$, InGaAs pin detectors indicated decent performance for near and mid-infrared applications. InGaAs pin detectors peaks at $1.75 \mu \mathrm{m}$ and with extended range, peaks at 1.95 and $2.3 \mu \mathrm{m}$. The corresponding $\mathrm{D}^{*}$ is $2.5,1.2$ and $0.2 \times 10^{12} \mathrm{cmHz}^{1 / 2} / \mathrm{W}$, at peak wavelength while cooling down to $-20^{\circ} \mathrm{C}^{4,5}$. Also InGaAs detectors are commercially available in the form of two-dimensional and linear arrays and avalanche photodiodes (APD) ${ }^{6,7}$. A shortcoming of InGaAs APD arises due to the shorter cut-off wavelength of $1.8 \mu \mathrm{m}$. On the other hand, InGaSb ternary material indicated good performance for $2 \mu \mathrm{m}$ detectors ${ }^{8}$, but still they are on the research level not being commercially available. The availability of ternary InGaSb virtual substrates has a promising potential for developing high performance detectors at wavelengths around the $2 \mu \mathrm{m}$, without the influence of the binary substrates usually used for processing the ternary materials ${ }^{9}$. 
Quaternary III-V compound materials are excellent candidate for $2 \mu \mathrm{m}$ detection. Several articles reported different device structures using materials such as $\mathrm{InGaAsSb}$ and $\mathrm{AlGaAsSb}{ }^{10-16}$. Several reports discussed the performance of InGaAsSb APD especially for applications such as optical communication ${ }^{10-14}$. Such devices usually involve complicated structures and sophisticated growth and processing procedures. Recently, using the same material, a new InGaAsSb/AlGaAsSb phototransistor has been developed ${ }^{15-17}$. Aside from an APD, a phototransistor can achieve higher gain and better signal-to-noise ratio, without the excess noise effects, which makes it attractive for $2 \mu \mathrm{m}$ applications. The performance of these devices has even exceeded the state-of-the-art InGaAs pin detector technology for some applications ${ }^{15-18}$. In the following sections we will present the characterization results for a phototransistor sample (A1-b10), with a special focus on measuring the device linearity and transient response.

\section{PHOTOTRANSISTOR CHARACTERISTICS}

The phototransistor studied in this work is a two terminal device, with a sensitive area diameter of $200 \mu \mathrm{m}$ and total mesa diameter of $400 \mu \mathrm{m}$. The device structure includes an n-type AlGaAsSb emitter, p-type composite base consisting of $\mathrm{AlGaAsSb}$ and InGaAsSb layers, and an n-type InGaAsSb collector ${ }^{17}$, ${ }^{18}$. Although, the detailed characterization of similar devices is presented elsewhere, the main performance results are presented in this section.

Figure 1(a) shows the dark current variation with bias voltage for the phototransistor sample at different temperatures. The dark current was obtained by measuring the device I-V characteristics in dark conditions and temperature controlled environment. The I-V resolution is $10 \mathrm{mV}$ with $10 \mathrm{~mA}$ maximum current limit or $4 \mathrm{~V}$ maximum reverse bias voltage. The device is characterized with relatively high dark current. I-V results point out the temperature dependence of the dark current on the bias voltage, and the absence of any avalanche gain, since there is no intersection in the characteristics, which confirms the transistor action. The temperature dependence of the dark current could be attributed to the nature of the dark current mechanism dominating each region. At low bias voltage, diffusion and generation-recombination currents are dominating the dark current and both of these components are highly temperature dependent. At higher bias voltage the tunneling current becomes dominant, which has much weaker temperature dependence ${ }^{19}$.

The operation of the phototransistor is associated with relatively high noise, which is mainly due to the high dark current of the device. The variation of the device noise current spectral density is presented in figure 1(b). The noise current was measured, using a spectrum analyzer (Stanford Research Systems; SR785) in the frequency band $0 \mathrm{~Hz}$ to $100 \mathrm{kHz}$, with $1-\mathrm{Hz}$ normalization and $50-\mathrm{kHz}$ center frequency. The phototransistor noise increases rapidly with increasing the device temperature and bias voltage. The profile of the noise current variation with bias voltage resembles the dark current profile. Cooling down the phototransistor reduces the noise, which is also proportional to the dark current variation with temperature observed in figure 1(a). The high noise of the device is compensated by the high gain, as presented, which leads to enhanced signal-to-noise ratio.

Figure 2(a) shows the quantum efficiency variation with wavelength at four different temperatures and zero bias voltage. Two distinctive regions appear in the quantum efficiency curve: a peak around the $2-\mu \mathrm{m}$ wavelength and a flat region between 1.2 and $1.8 \mu \mathrm{m}$. This profile corresponds mainly to the absorption in the InGaAsSb region and the structure of the device. Cooling the device shifts the cutoff wavelength to a shorter value, corresponding to the change of the absorption edge with temperature. The location of the maximum quantum efficiency always occurs at $2 \mu \mathrm{m}$, independent on the temperature. For a specific wavelength, an optimum operating temperature can be defined by observing the quantum efficiency variation with temperature, as indicated in figure 2(b). The optimum temperature that maximizes the quantum efficiency changes with the operating wavelength. As indicated in the figure this temperature is equal to $-20,30$ and $60^{\circ} \mathrm{C}$ for 2050,1540 and $1065 \mathrm{~nm}$, respectively.

The spectral response of a detector defines its responsivity variation with the wavelength of the incident radiation. To achieve the spectral response, the device was calibrated using the substitution method, in reference to a calibrated $3 \times 3 \mathrm{~mm}^{2} \mathrm{PbS}$ detector ${ }^{20,21}$. Figure 3(a) shows the spectral response calibration results for the phototransistor, with a 20 $\mathrm{nm}$ resolution. Cooling down the device reduces the dark current, which allows for increasing the applied bias voltage. This results in higher gain and responsivity. On the other hand, further cooling of the device leads to the loss of the sensitivity at longer wavelength, due to the shift in the absorption edge, as indicated in the quantum efficiency results. 
Figure 3(b) shows the responsivity variation with bias voltage and temperature. It is interesting to observe that the responsivity increases by increasing the temperature at a fixed bias voltage. Since the quantum efficiency decreases at higher temperature, as shown in figure 2(b), this indicates a higher gain of the device at higher temperatures. For example: at $2.05 \mu \mathrm{m}, 100^{\circ} \mathrm{C}$ and $2 \mathrm{~V}$ the device gain is 154.3 , which correspond to $94.7 \mathrm{~A} / \mathrm{W}$ responsivity and $9.8 \times 10^{-10}$ $\mathrm{A} / \mathrm{Hz}^{1 / 2}$ noise current. The resultant noise-equivalent-power (NEP) is about $1 \times 10^{-11} \mathrm{~W} / \mathrm{Hz}^{1 / 2}$ equivalent to $1.7 \times 10^{9}$ $\mathrm{cmHz}^{1 / 2} / \mathrm{W}$. Reducing the temperature to $-30^{\circ} \mathrm{C}$ and increasing the bias to $3 \mathrm{~V}$ the device gain becomes 231.8 . This corresponds to $318.6 \mathrm{~A} / \mathrm{W}$ responsivity and $8.6 \times 10^{-11} \mathrm{~A} / \mathrm{Hz}^{1 / 2}$ noise current, resulting in NEP of about $2.7 \times 10^{-13} \mathrm{~W} / \mathrm{Hz}^{1 / 2}$ equivalent to $6.5 \times 10^{10} \mathrm{cmHz}^{1 / 2} / \mathrm{W}$.

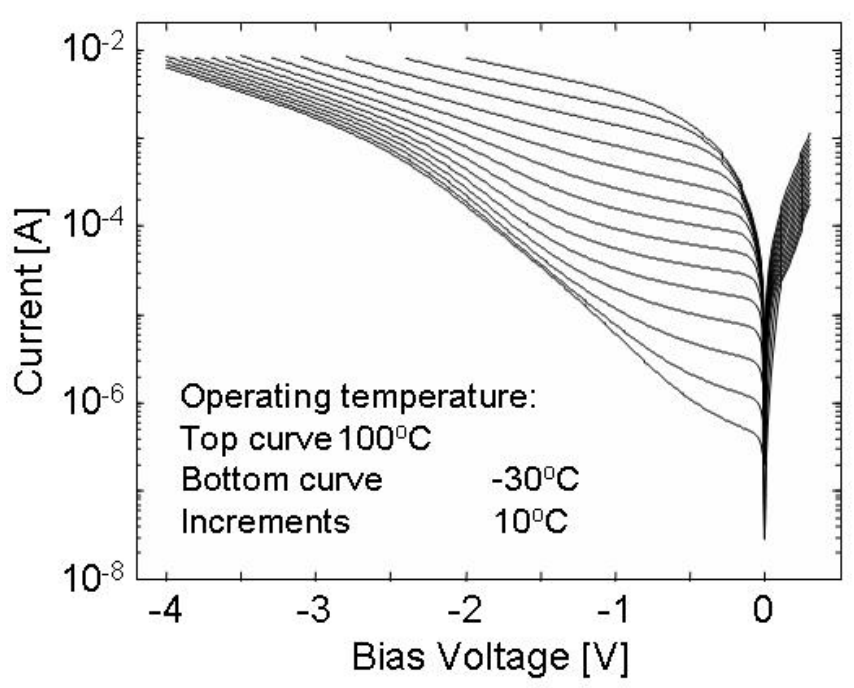

(a)

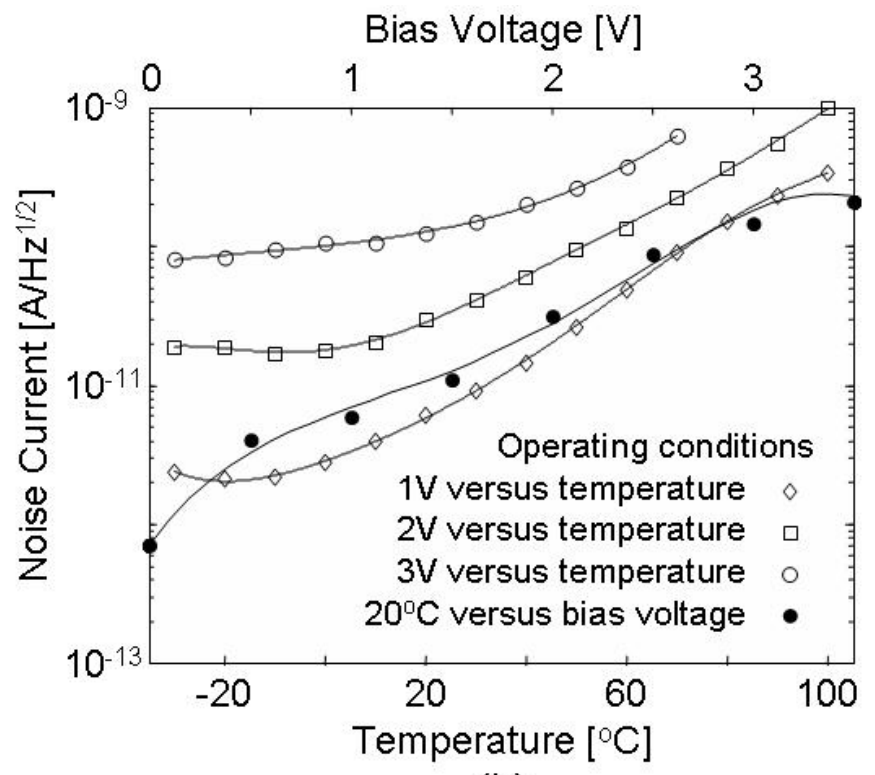

(b)

Figure 1 (a) Phototransistor I-V characteristics at different temperatures, and (b) noise current spectral density variation with bias voltage at $20 \mathrm{oC}$ and with temperature at the specified bias voltages.

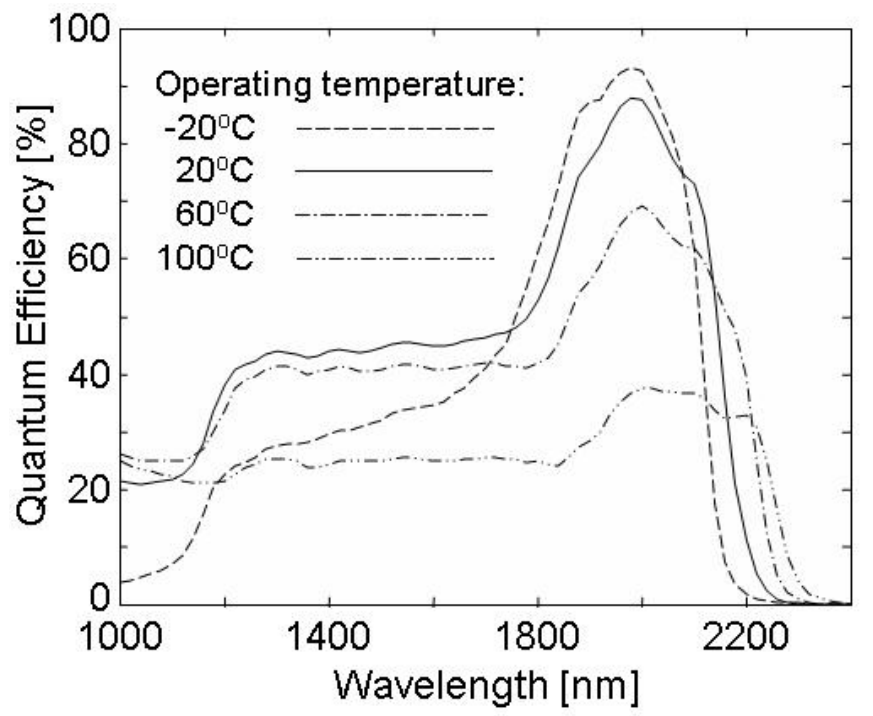

(a)

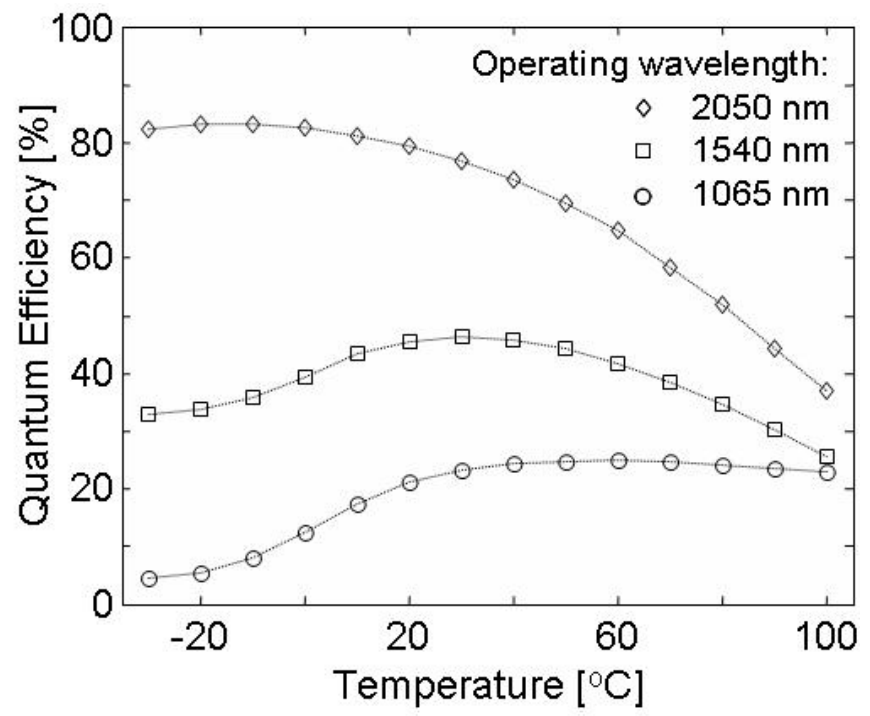

(b)

Figure 2 (a) Zero-bias quantum efficiency variation with wavelength at selected temperatures and (b) the corresponding quantum efficiency variation with temperature for selected wavelength. 


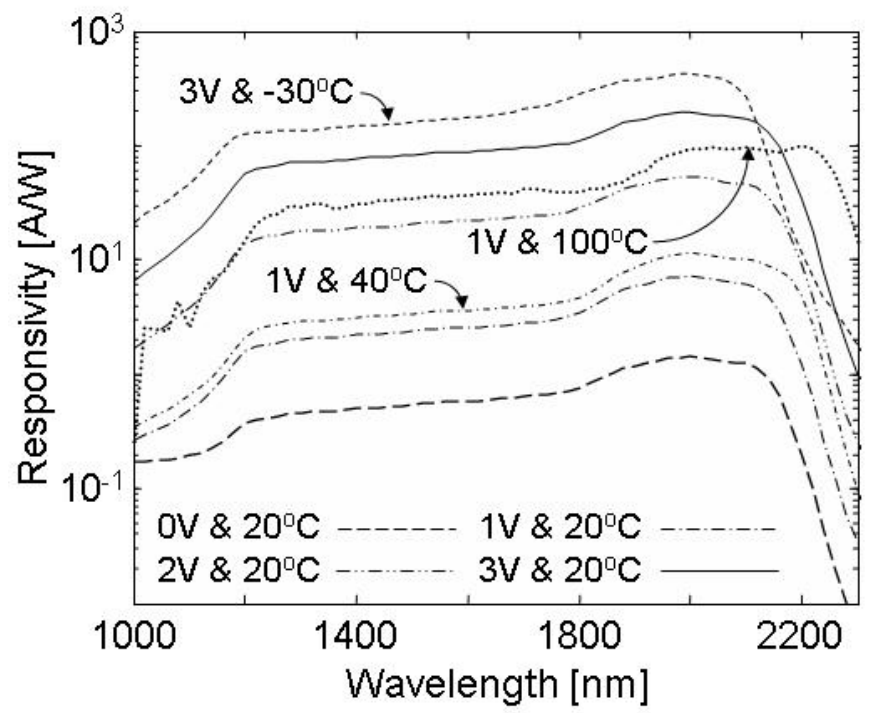

(a)

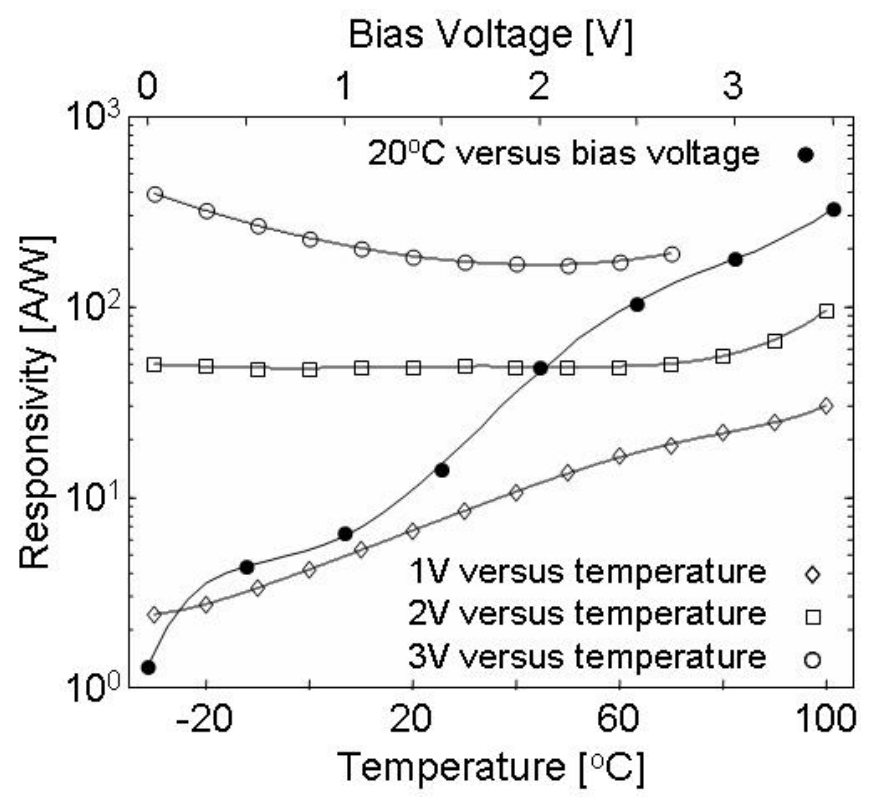

(b)

Figure 3 (a) The phototransistor spectral response at different operating conditions and (b) the responsivity variation with bias voltage and temperature at $2.05 \mu \mathrm{m}$ and selected operating conditions.

\section{PHOTOTRANSISTOR LINEARITY}

Ideally, the responsivity of an optical detector is a predetermined quantity that depends on the device bias voltage, temperature and the wavelength of the incident radiation, and is independent on the incident radiation intensity. This is true for a real device, within an incident intensity range, defining the device dynamic range. Intensity above this range starts to saturate the device, while the lowest intensity range is determined by the device NEP. Therefore, the linearity of an optical detector defines how fixed the responsivity within the dynamic range of the device. In this section, the linearity of the InGaAsSb/AlGaAsSb phototransistor is characterized. Figure 4 shows the characterization setup for measuring the device dynamic range. The setup consists of an optical, electrical and detector control sections. The optical section consists of a CW $2 \mu \mathrm{m}$ laser source, the output of which is filtered and modulated. Filtering starts with $2.05 \mu \mathrm{m}$ line filter then a set of a combinational neutral density filters. The detector is placed in a detector chamber, which controls the device temperature using a temperature controller, which controls a thermoelectric cooler with a thermistor feedback element. For temperature operation lower than $0^{\circ} \mathrm{C}$, nitrogen is purged to prevent water condensation and ice formation on the detector, while a chiller is used to dump the excess heat. In the electrical section, the detector output is converted to a voltage signal using a preamplifier the output of which is measured using a lock-in amplifier, synchronized to the chopper controller.

Generally, using this setup, the intensity of the radiation source can be varied through a wide intensity level (about 7 orders of magnitude). The intensity can be changed either by controlling the laser driving current, neutral density filter setting or by the distance between the detector and the source. With a certain setting, the intensity is measured using a calibrated InGaAs pin detector. Then the test phototransistor is placed in the same location, using an optical microscope, while measuring its output. Comparing the reading of the lock-in amplifier with an oscilloscope, the rms lock-in reading is converted to a peak-to-peak reading by considering the chopping factor. Figure 5 shows the output current variation with the incident radiation intensity at various operating conditions. At a certain bias voltage and temperature, corresponding to a constant responsivity, the phototransistor current increases with the incident intensity. It is clear from the figure that the device is linear up to an intensity level of $31.6 \mathrm{~mW} / \mathrm{cm}^{2}$. Although the minimum detectable intensity should be dependent on the device NEP, the $1.6 \times 10^{-7} \mathrm{~W} / \mathrm{cm}^{2}$ level (minimum intensity shown in figure 5) is attributed to the setup limitation due to the InGaAs pin detector. The phototransistor dynamic rage corresponding to the results shown in the figure is equal to $100 \mathrm{~dB}$. 


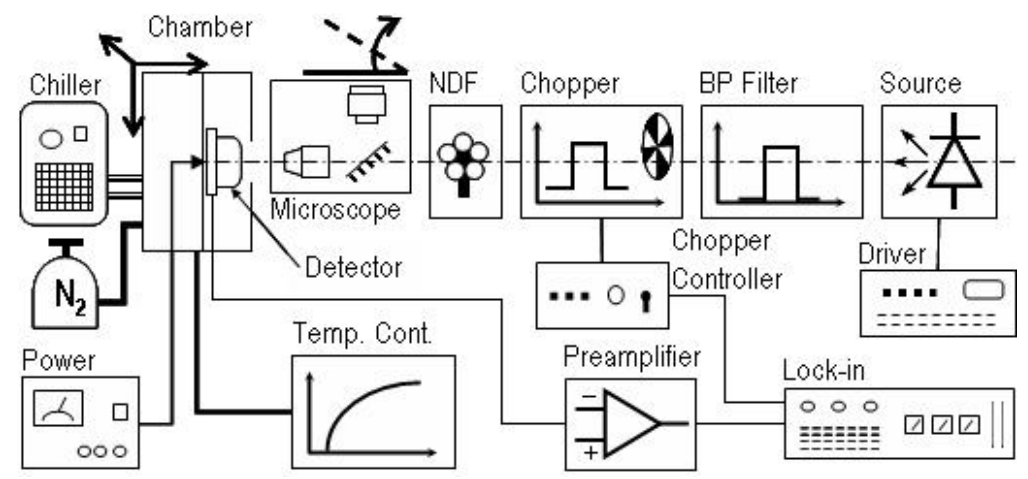

Figure 4 Experimental setup for measuring the detector linearity and dynamic range.

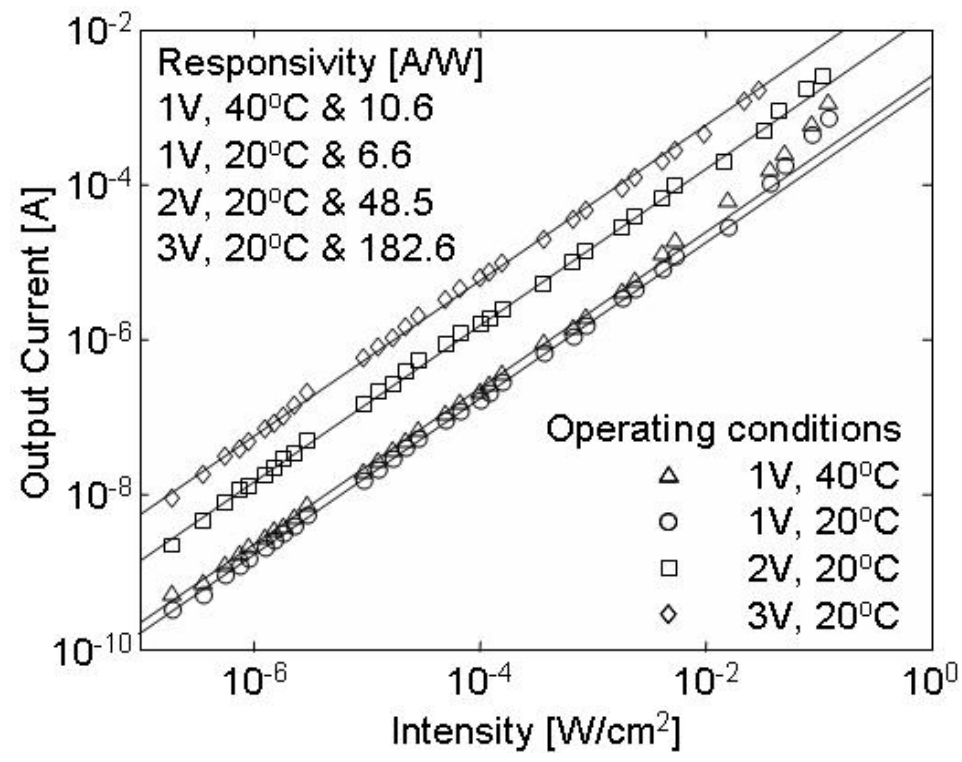

Figure 5 Variation of the phototransistor output current with applied intensity at $2.05 \mu \mathrm{m}$, compared to constant responsivity contours, at the specified operating conditions.

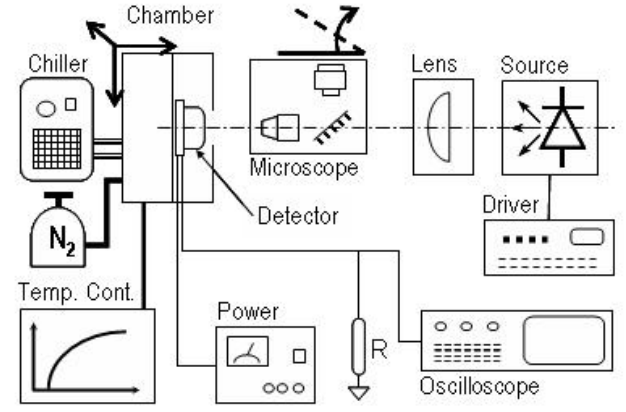

(a)

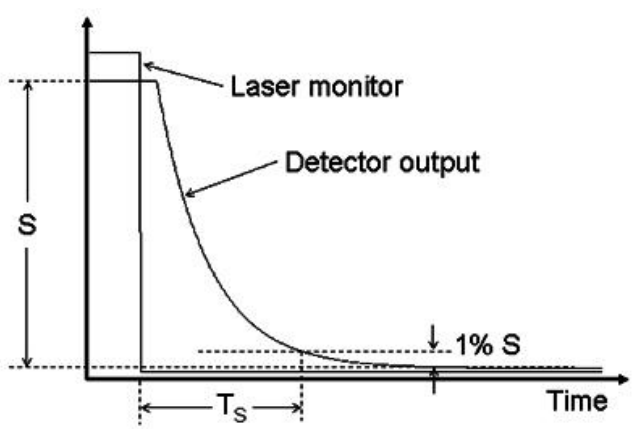

(b)

Figure 6 (a) Experimental setup for measuring the detector impulse response. (b) Definition of the settling time with respect to the laser monitor and the detector output. 


\section{PHOTOTRANSISTOR IMPULSE RESPONSE}

The impulse response of the phototransistor was characterized to examine the transient behavior of the device. In some applications, such as remote sensing using the lidar technique, the detector is subjected to a severe optical signal generated from the atmospheric backscattering. In such conditions, the near-field backscattering signal has a very high intensity that could saturate the detector, whereas the far-field signal has a weak intensity that is close to the detection limit of the device. The detector should be able to totally recover the near-field signal, in a relatively short time, so there will be a minimal influence to the far field signal. This recovery time is directly proportional to the settling time of the whole detection system including the settling time of the detector. Therefore the phototransistor settling time is a very important parameter that influences the whole lidar instrument.

The phototransistor settling time is characterized using the setup shown in figure 6(a). Similarly, the setup consists of an optical, electrical and detector control sections. The optical section consists of a $2 \mu \mathrm{m}$ pulsed laser, with a pulse duration of $1 \mu \mathrm{s}$ and repetition rate of $16 \mathrm{kHz}$ with a built-in monitor. The laser current is adjusted to apply 8.75 $\mathrm{mWatt} / \mathrm{cm}^{2}$ radiation intensity at the detector surface. The laser radiation is collimated using a lens and applied to the detector sensitive area. The microscope is used to define the optical axis and align the detector to the same distance relative to the radiation source. The detector is placed in the detector chamber to control its temperature and provide a mechanical support for alignment. The detector output is directly connected to a power supply for biasing and terminated to a $50 \Omega$ resistor. The $50 \Omega$ termination acts as a current-to-voltage converter, which is connected to the input of an oscilloscope. This connection avoids the use of a transimpedance amplifier, which might influence the detector transient response. Figure 6(b) shows a typical output of the detector, compared to the output of the laser monitor. Due to the equivalent impedance of the detector, the output resembles an exponential decay function, with a time constant mainly dependent on the device capacitance and the termination resistance. Aside of the conventional definition of the settling time (the time interval for the signal to change from $90 \%$ and $10 \%$ of the amplitude), figure $6(\mathrm{~b})$ defines the settling time according to our measurements. In this case the settling time is the time interval that starts by the falling edge of the monitor signal and ends by the instant the detector output signal reach $1 \%$ of the amplitude. Figure 7 (a) shows the variation of the settling time with the bias voltage obtained at $20^{\circ} \mathrm{C}$. Increasing the bias voltage increases the settling time. This behavior is well understood if we assume a constant gain-bandwidth product for the phototransistor. Increasing the bias voltage increases the phototransistor gain which leads to reduce the bandwidth resulting in a slower device, i.e. longer settling time. Figure 7(b) shows the variation of the settling time with temperature, obtained at three different bias voltage levels. For a certain bias, the settling time decreases with increasing the temperature. This might be attributed to the recombination processes (recombination lifetime), which is affected by the temperature. At higher temperature the recombination of the photo generated charge carriers is much faster, leading to a shorter time constant, i.e. faster settling time.
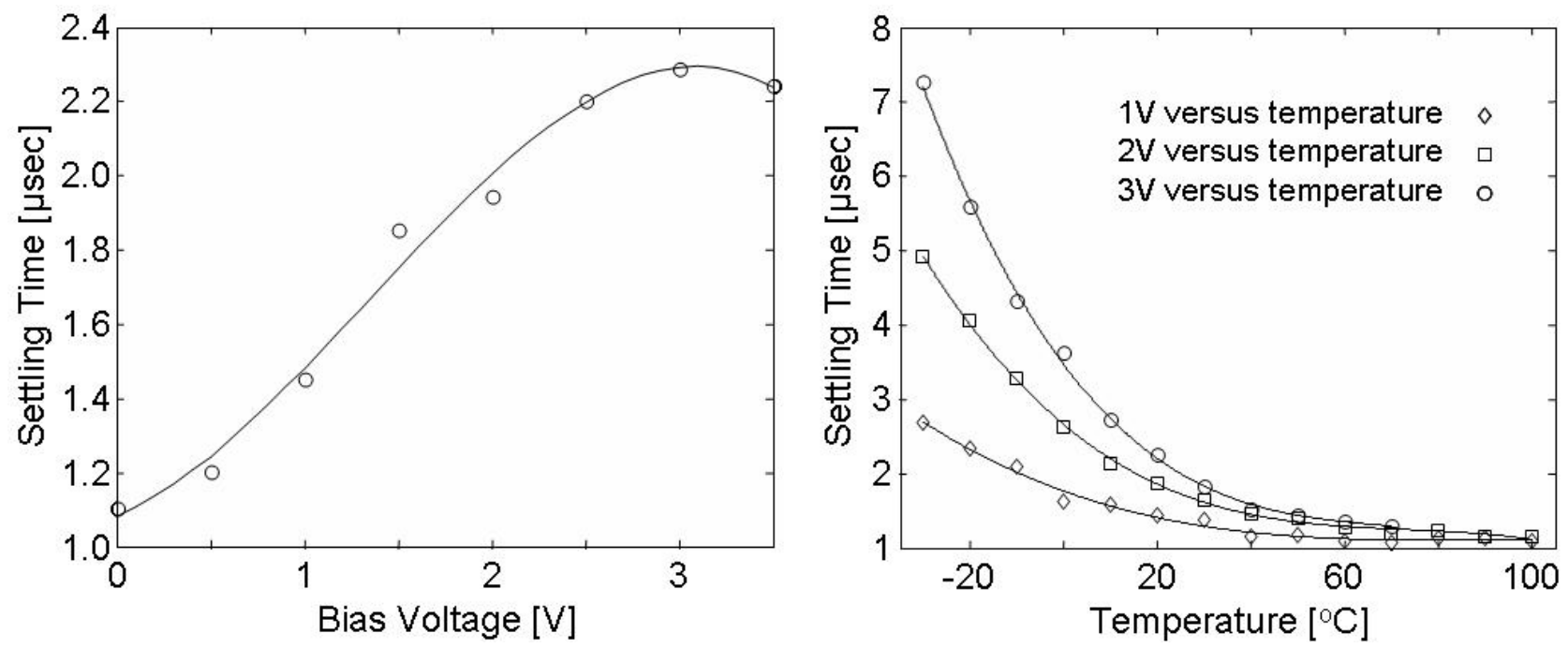

Figure 7 (a) Settling time variation with bias voltage at $20^{\circ} \mathrm{C}$ and (b) variation with temperature at listed voltages, obtained at $8.75 \mathrm{mWatt} / \mathrm{cm}^{2}$ radiation intensity at $2 \mu \mathrm{m}$ wavelength. 


\section{CONCLUSION}

In this paper, the characteristics of a novel npn InGaAsSb/AlGaAsSb heterojunction phototransistor is presented. Optimized to the $2 \mu \mathrm{m}$ wavelength, with a sensitivity from $1 \mu \mathrm{m}$ to $2.3 \mu \mathrm{m}$, these phototransistors show strong potential for developing IR sensors with improved performance. The performance of the device is presented and includes dark current, noise, spectral response and quantum efficiency measurements. The new phototransistor survives high temperature deviations. The phototransistor sample was tested at temperatures up to $100^{\circ} \mathrm{C}$ and down to $-30^{\circ} \mathrm{C}$ and the results were reproducible, without device damage. This indicates the potential of these devices for severe operating conditions. Focusing on $2.05 \mu \mathrm{m}$ wavelength, at $100^{\circ} \mathrm{C}$ and $2 \mathrm{~V}$ the device gain is 154.3 , which correspond to $94.7 \mathrm{~A} / \mathrm{W}$ responsivity and $9.8 \times 10^{-10} \mathrm{~A} / \mathrm{Hz}^{1 / 2}$ noise current. The resultant NEP is about $1 \times 10^{-11} \mathrm{~W} / \mathrm{Hz}^{1 / 2}$ equivalent to $1.7 \times 10^{9}$ $\mathrm{cmHz}^{1 / 2} / \mathrm{W}$. Reducing the temperature to $-30^{\circ} \mathrm{C}$ and increasing the bias to $3 \mathrm{~V}$ the device gain becomes 231.8 . This corresponds to $318.6 \mathrm{~A} / \mathrm{W}$ responsivity and $8.6 \times 10^{-11} \mathrm{~A} / \mathrm{Hz}^{1 / 2}$ noise current, resulting in NEP of about $2.7 \times 10^{-13} \mathrm{~W} / \mathrm{Hz}^{1 / 2}$ equivalent to $6.5 \times 10^{10} \mathrm{cmHz}^{1 / 2} / \mathrm{W}$. Dynamic range measurements indicated that the device is linear between the intensity levels of $1.6 \times 10^{-7} \mathrm{~W} / \mathrm{cm}^{2}$ and $3.2 \times 10^{-2} \mathrm{~W} / \mathrm{cm}^{2}$. Impulse response measurements indicated a room temperature settling time of about $2 \mu \mathrm{s}$ at $20^{\circ} \mathrm{C}$ and $2 \mathrm{~V}$. Increasing the temperature to $100^{\circ} \mathrm{C}$ leads to reduce the settling time to $1.1 \mu \mathrm{s}$.

\section{ACKNOWLEDGMENT}

This effort is part of the Laser Risk Reduction Program funded by NASA's Earth Science Technology Office and the Enabling Concepts and Technology Program within NASA’s Aerospace Technology Enterprise.

\section{References}

1. I. Bhat, Y. Xiao, V. Bhagwath, P. Dutta, T.F. Refaat and M.N. Abedin, "GaInAsSb-based IR detectors in the 2 $\mu \mathrm{m}$ range fabricated from layer structures grown by metalorganic vapor phase epitaxy", Military Sensing Symposia MSS, 2005.

2. J12D Series InAs Detectors Operating Instructions, Judson Technologies, PB220, October, 2000.

3. J10D Series InSb Detectors Operating Instructions, Judson Technologies, PB211, August, 2000.

4. InGaAs PIN photodiode G8422/G8372/G5852 series, Data Sheet, Hamamatsu, October, 2004.

5. InGaAs PIN photodiode G8423/G8373/G5853 series, Data Sheet, Hamamatsu, March, 2005.

6. $\quad$ C30645E/C30662E Series InGaAs Avalanche Photodiode, Data Sheet, PerkinElmer, 2004.

7. T. Martin and P. Dixon, "InGaAs sees infrared and visible light", Laser Focus World, 40(11), 2004.

8. T.F. Refaat , M.N. Abedin, I. Bhat, P. Dutta and U.N. Singh, "Characterization of InGaSb/GaSb p-n photodetectors in the 1.0- to 2.4- $\mu \mathrm{m}$ wavelength range", Optical Engineering, 43(5), 1014-1015, 2004.

9. T.F. Refaat, M.N. Abedin, V. Bhagwat, I. Bhat, P. Dutta and U.N. Singh, "InGaSb photodetectors using an InGaSb substrate for 2- $\mu$ m applications", Applied Physics Letters, 85(11), 1874-1876, 2004.

10. M. Mikhailova, I. Andreev, A. Baranov, S. Mel'nikov, Y. Smortchkova and Y. Yakovlev, "Low-noise GaInAsSb/GaAlAsSb SAM avalanche photodiode in the 1.6-2.5 $\mu \mathrm{m}$ spectral range", Proceedings of SPIE, 1580, 308-312, 1991.

11. I. Andreev, M. Afrailov, A. Baranov, M. Mirsagatov, M. Mikhailov, and Y. Yakovlev, "GaInAsSb/GaAlAsSb avalanche photodiode with separate absorption and multiplication regions. Soviet Technical Physics Letters, 435437, 1988.

12. J. Benoit, M. Boulou, G. Soulage, A. Joullie, and H. Mani, "Performance evaluation of GaAlAsSb/GaInAsSb SAM-APDs for high bit rate transmission in the $2.5 \mu \mathrm{m}$ wavelength region", Journal of Optical Communication, 9(2), 55-58, 1988.

13. I. Andreev, M. Afrailov, A. Baranov, S. Konnikov, M. Mirsagatov, M. Mikhailova, O. Salata, V. Umanskii, G. Filaretova, and Y. Yakovlev, "Ultrafast GaInAsSb p-i-n photodiode for the spectral interval 1.5-2.3 $\mu$ m", Soviet Technical Physics Letters, 15(4), 253-254, 1989.

14. O.V. Sulima, M.G. Mauk, Z.A. Shellenbarger, J.A. Cox, P.E. Sims, S. Datta, and S.B. Rafol, "Uncooled lowvoltage AlGaAsSb/InGaAsSb/GaSb avalanche photodetectors", IEE Proceedings - Optoelectronics, 151(1), 1-5, 2004. 
15. T.F. Refaat, M.N. Abedin, O.V. Sulima, S. Ismail and U.N. Singh, "AlGaAsSb/InGaAsSb phototransistors for 2$\mu \mathrm{m}$ remote sensing applications", Optical Engineering, 43(7), 1647-1650, 2004.

16. O.V. Sulima, T.F. Refaat, M.G. Mauk, J.A. Cox, J. Li, S.K. Lohokare, M.N. Abedin, U.N. Singh and J.A. Rand, "AlGaAsSb/InGaAsSb phototransistors for spectral range around $2 \mu \mathrm{m}$ ", Electronics Letters, 40(12), 766-767, 2004.

17. M.N. Abedin, T.F. Refaat, O.V. Sulima and U.N. Singh, "AlGaAsSb/InGaAsSb heterojunction phototransistor with high optical gain and wide dynamic range", IEEE Transaction on Electron Devices, 51(12), 2013-2018, 2004.

18. T.F. Refaat, M.N. Abedin, O.V. Sulima, S. Ismail and U.N. Singh, "III-V compound detectors for $\mathrm{CO}_{2}$ DIAL measurements", (Invited) Lidar Remote Sensing for Environmental Monitoring VI; Proceedings of SPIE, Vol. 5887, 588706, 2005.

19. S. Forrest, "Performance of InGaAsP photodiodes with dark current limited by diffusion, generation, recombination and tunneling”, IEEE Journal of quantum Electronics, QE-17(2), 217-226, 1981.

20. M.N. Abedin, T.F. Refaat, O.V. Sulima and U.N. Singh, "Recent development of Sb-based phototransistors in the 0.9- to $2.2 \mu \mathrm{m}$ wavelength range for applications to laser remote sensing", Workshop on Frontiers in Electronics (WOFE-04), 2004.

21. T. Larason, S. Bruce, and A. Parr, "Spectroradiometric detector measurements: part I ultraviolet detectors and part II visible to near-infrared detectors", NIST Special Publications, 250- 41, 1998. 